\title{
Incidence and Clinical Management of Atrial Arrhythmias in Patients with Catecholaminergic Polymorphic Ventricular Tachycardia
}

\author{
Gurukripa Kowlgi $^{1}$, John Giudicessi ${ }^{2}$, Walid Barake ${ }^{1}$, Konstantinos Siontis ${ }^{1}$, Johan Bos ${ }^{3}$, \\ and Michael Ackerman ${ }^{1}$ \\ ${ }^{1}$ Mayo Clinic \\ ${ }^{2}$ Mayo Clinic Minnesota \\ ${ }^{3}$ Mayo CLinic
}

September 25, 2021

\begin{abstract}
Background: Catecholaminergic polymorphic ventricular tachycardia (CPVT) is a genetic arrhythmia syndrome characterized by adrenergically-triggered ventricular arrhythmias, syncope, and sudden cardiac death. Several small studies suggest that atrial arrhythmias (AAs) are common in patients with CPVT. Objective: To determine the incidence and type of AAs observed within a large, single-center cohort of CPVT cases as well as the efficacy and durability of AA-directed management. Methods: In this retrospective study, the electronic medical record of 129 patients ( $52 \%$ female; average age at diagnosis 20.8 ? 15.3 years) with CPVT (95\% with a putative CPVT1-causative RYR2 variant) between 01/2000 and 09/2019 were reviewed for electrocardiographic evidence of AAs. Clinical features and efficacy of pharmacologic and ablation therapy were assessed. Results: Overall, 10/129 (7.8\%) CPVT patients, all RYR2 variant-positive, had evidence of an AA (atrial fibrillation/flutter in 6 , atrial tachycardia in 3, and supraventricular tachycardia in 1). The median age at AA diagnosis was 23 (14.2-35.5) years. $8 / 10$ of patients experienced symptoms attributed to their AA, including inappropriate shocks. All patients were trialed on anti-arrhythmics, including ?-blockers, and/or flecainide. Owing to drug failure (1/10), drug intolerance (1/10), or patient preference $(2 / 10) ; 4 / 10$ patients received an ablation. Over a median follow-up of 23.5 (4.5-63) months, no AA recurrences were observed. Conclusion: Compared to prior studies, the incidence of AAs in this large, single-center referral cohort of CPVT patients was substantially lower ( $7.8 \%$ vs. $26 \%-35 \%)$. Although larger multi-center studies are needed to confirm, this study suggests that ablation is efficacious and durable in CPVT-associated AAs.
\end{abstract}

\section{Hosted file}

2021 - JCE - AA in CPVT - Kowlgi - MANUSCRIPT.docx available at https://authorea.com/ users/338358/articles/538974-incidence-and-clinical-management-of-atrial-arrhythmiasin-patients-with-catecholaminergic-polymorphic-ventricular-tachycardia 